\title{
Volumetric reconstruction of the right ventricle improves reproducibility of interventricular septum bowing in patients with pulmonary embolism
}

\author{
G. Staśkiewicz ${ }^{1,2}$, E. Czekajska-Chehab², S. Uhlig², A. Tomaszewski ${ }^{3}$, K. Torres ${ }^{1}$, \\ A. Torres ${ }^{1}$, M. Walankiewicz ${ }^{1}$, W. Surtel ${ }^{4}$, A. Drop ${ }^{2}$ \\ ${ }^{1}$ Department of Human Anatomy, Medical University of Lublin, Lublin, Poland \\ $21^{\text {st }}$ Department of Radiology, Medical University of Lublin, Lublin, Poland \\ ${ }^{3}$ Department of Cardiology, Medical University of Lublin, Lublin, Poland \\ ${ }^{4}$ Department of Electronics, Lublin University of Technology, Lublin, Poland
}

[Received 2 November 2012; Accepted 12 January 2013]

Bowing of the interventricular septum (IVS) is a sign of severe pulmonary embolism (PE); however, it is affected by high interobserver variability. The aim of the study was to evaluate the application of volumetric reconstructions of the right ventricle for assessment of IVS position regarding its accuracy in identifying right ventricular dysfunction, as well as interobserver agreement in evaluating this sign. IVS bowing was evaluated with multiplanar reformations (MPR) and volumetric reconstruction (VR, volume rendering) by 2 observers. The accuracy of IVS bowing sign was better for VR-based assessment; however, it was not significantly better than the MPR-based evaluation. Interobserver agreement was found to be fair (kappa $=0.381$ ) for the MPR-based grading of IVS position as normal or abnormal, while it was significantly better for the VR method (kappa $=0.629$, $p<0.001)$. The VR-based method may improve utilisation of IVS bowing sign in patients with PE. (Folia Morphol 2013; 72, 2: 107-112)

Key words: pulmonary embolism, computed tomography, right ventricular dysfunction, interobserver agreement, volumetric reconstruction

\section{INTRODUCTION}

Acute pulmonary embolism (PE) is among the most common causes of cardiovascular mortality, preceded by acute coronary syndromes and stroke [6]. However, as the clinical symptoms and signs in patients with PE may mimic multiple cardiovascular or pulmonary conditions, imaging methods have become one of cornerstones in the diagnostic process in this group of patients, with computed tomography pulmonary angiography (CTPA) becoming the gold-standard in the imaging of suspected PE.
CTPA allows concurrent visualisation of both pulmonary arteries with the exact localisation of the obstruction site, as well as heart chambers and mediastinal vessels. Identification of right ventricular dysfunction (RVD) allows for a better risk stratification in patients with acute PE [17].

Bowing of the interventricular septum (IVS) is one of the signs of RVD that has been adapted from echocardiography; however, it is affected by high interobserver variability $[2,9]$. It is known to occur in cases of severe pulmonary artery systolic pressure 
(PASP) increase; therefore, it allows identification of patients at particularly high risk. The aim of the study was to evaluate the feasibility of volumetric reconstruction (VR, volume rendering) of the right ventricle for improved utilisation of this sign.

\section{MATERIALS AND METHODS}

The institutional ethical review board approved the protocol of the study with a waiver of informed consent - decision no: KE-0254/41/2011. For the purpose of this retrospective study, we identified 60 consecutive patients with CTPA confirmed acute $P E$, in whom echocardiographic examination with PASP assessment was performed within $12 \mathrm{~h}$ from CT scanning. The CTPA studies were performed with a 64-row VCT scanner (GE Medical Systems), with a standard protocol used at our institution, which includes craniocaudal scanning of the chest with collimation of $0.625 \mathrm{~mm}$, SmartPrep adjustment of injection time of $70 \mathrm{~mL}$ of iodinated contrast medium (Ultravist 370, Schering AG, Germany) administered with a flow rate of $4-5 \mathrm{~mL} / \mathrm{s}$, followed by injection of $40 \mathrm{~mL} \mathrm{NaCl}$ bolus.

Advantage Workstation 4.3 (GE Healthcare Systems) was used for processing of the examinations, which were reviewed independently by 2 readers with 7 years and 5 years of experience with cardiothoracic $\mathrm{CT}$ analysis. The image settings were individually adjusted by the readers to optimise the display of pulmonary arteries and cardiac chambers. The readers were blinded to PASP values.

Pulmonary arteries obstruction was scored according to Qanadli et al. [14]. The maximum short axes of the right (RV) and left ventricles (LV) were measured in a 4 chamber view, according to Quiroz et al. [15]. The position of the IVS was evaluated and classified as normal (convex toward the RV), flattened, or convex toward LV. VR reconstructions of the RV were prepared on the same workstation. A volumetric analysis session was performed two weeks after the primary analysis and included visual assessment of the medial wall of the RV, visualised by means of VR. The readers were allowed to rotate the volumetric reconstructions to best visualise the spatial configuration of the medial wall of the RV. No additional measurements were taken during the volumetric analysis session.

An interrater reliability analysis using the kappa statistic was performed to determine the consistency among observers for multiplanar and volumetric identification of IVS position. The kappa values were interpreted as follows: poor: $\leq 0.20$; fair: $0.21-0.40$; moderate: $0.41-0.60$; good: $0.61-0.80$; and very good: $\geq 0.81$ [1]. Interobserver agreement analyses were performed with SPSS 16.0 for Windows. ROC curves for multiplanar reformations (MPR)- and VR-based abnormal position of IVS for identification of increased PASP values were constructed and compared with Aabel 3.0 statistical package. To illustrate the 3-dimensional configuration of IVS for publication purposes, stereoscopic views of the medial wall of the RV were prepared with Amira 5 medical image analysis software. Stereoscopic images were not used for the diagnosis of IVS bowing, where the observers used VR reconstructions.

\section{RESULTS}

The study group consisted of 60 patients, median age 71 (range 26-89) years. Median Qanadli score was 18 (range 2-38) points, and the RV/LV ratio was 1.13 (0.7-2.19). Median PASP value was 40 (26-70) $\mathrm{mm} \mathrm{Hg}$. Two patients died in the 30-day period following the PE diagnosis.

Overall interobserver agreement for describing the IVS position by means of MPR reconstructions was fair: for classification as normal, flattened or bowed was it was 0.333 , while for identification of normal vs. abnormal configuration of IVS it was 0.381 . Visual assessment of the medial wall of the RV by means of volume rendering reconstruction was characterised by good interobserver agreement, 0.559 and 0.629 , respectively. MPR and VR-based visualisation of IVS are presented in Figures 1 and 2. Detailed results of both grading methods are presented in Table 1 .

The position of the IVS was compared with PASP values for the MPR and VR methods by both observers. VR assessment was characterised by a larger area under the ROC curve, which shows its higher accuracy as compared with the MPR-based identification (Fig. 3); however, the difference of areas was not statistically significant. Furthermore, obstruction score, RV/LV ratio, and PASP values were compared between patients with normal and abnormal position of IVS in 3-dimensional assessment, VR(-) and VR(+) groups, respectively, to show the reliability of this visualisation method. The accuracy of the IVS position assessment with MPR-based method for observers 1 and 2 was $72 \%$ and $64 \%$, respectively, and for VR-based method it was $65 \%$ and $68 \%$, respectively. Areas under the ROC curve (AUC), and the specificity and sensitivity of the above-mentioned parameters 


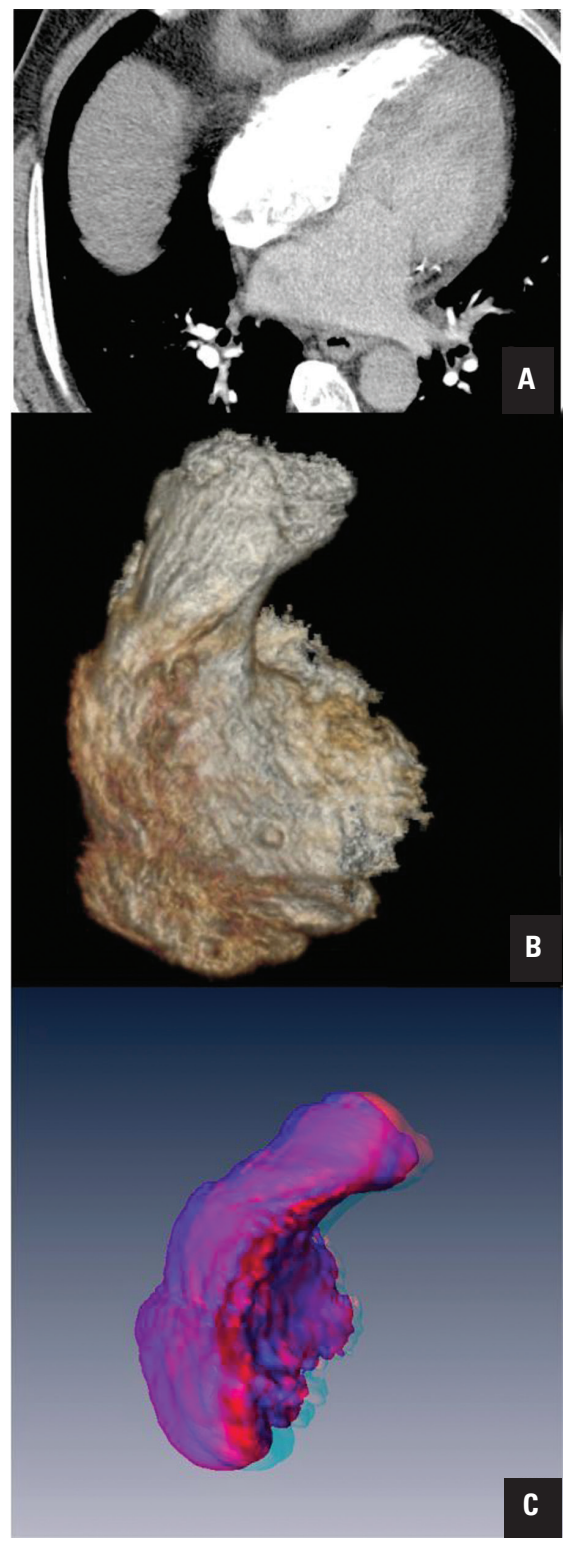

Figure 1. Patient with interventricular septum position graded as normal by both observers, with both multiplanar reformation (MPR)- and volume rendering (VR)-based method; A. MPR 4-chamber view; $\mathbf{B}$. VR reconstruction, used for grading the position of medial wall of right ventricle; C. Stereoscopic reconstruction of the right ventricle.

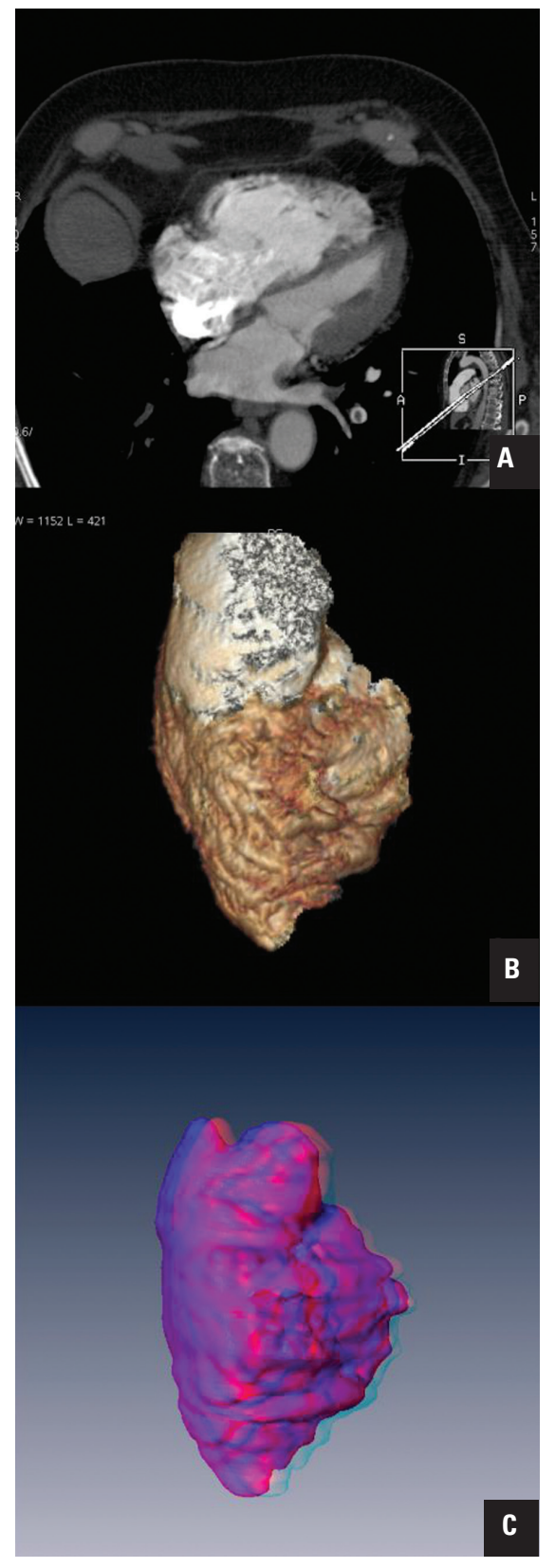

Figure 2. Patient with interventricular septum position graded as left-sided shift by both observers, with both multiplanar reformation (MPR)and volume rendering (VR)-based method; A. MPR 4-chamber view; B. VR reconstruction, used for grading the position of the medial wall of the right ventricle; C. Stereoscopic reconstruction of the right ventricle.

Table 1. Identification of interventricular septum position and interobserver agreement of the multiplanar reformations (MPR) and volumetric method (VR, volume rendering)

\begin{tabular}{ccccccc}
\hline & Normal & Flattened & Bowing & $\begin{array}{c}\text { Normal vs. abnormal } \\
\text { Kappa [95\% Cl] }\end{array}$ & $\begin{array}{c}\text { Normal vs. flattened vs. bowing } \\
\text { Kappa [95\% Cl] }\end{array}$ \\
\hline \multirow{2}{*}{ MPR } & Observer 1 & 39 & 16 & 5 & $0.381[0.268-0.495]$ & $0.333[0.232-0.434]$ \\
& Observer 2 & 33 & 18 & 9 & & \\
\multirow{2}{*}{ VR } & Observer 1 & 35 & 23 & 2 & $0.629[0.530-0.729]$ & $0.559[0.459-0.658]$ \\
& Observer 2 & 43 & 14 & 3 & & \\
\hline
\end{tabular}




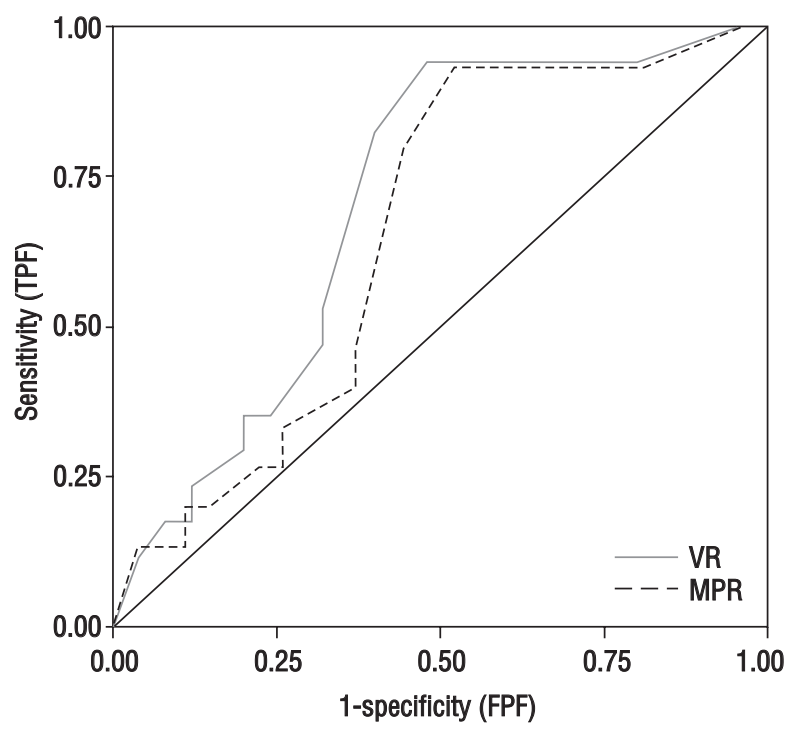

Figure 3. Comparison of ROC curves for multiplanar reformation (MPR)- and volume rendering (VR)-based assessment of normal vs. abnormal position of interventricular septum.

were calculated with regard to IVS bowing and are presented in Table 2.

\section{DISCUSSION}

Pulmonary hypertension and RV overload may occur in the course of acute $\mathrm{PE}$, as a result of both mechanical and humoral effects of pulmonary obstruction. The RV is characterised by a different structure than the left one, including its wall thickness, contractility, and tolerance of after- and preload changes. Its geometry makes it poorly tolerant to acute changes of afterload. Configuration of the IVS changes with the increase of RV pressure in patients with severe pulmonary obstruction [8], as a result of elevated RV pressure and increased RV volume. As the heart is covered by inelastic pericardium, changes in RV pressure and size (which may be accompanied by acute tricuspid regurgitation), as well as IVS configuration affect LV:
LV size and compliance are decreased, which reduces the LV preload and stroke volume, which may result in systemic hypotension. Echocardiographic assessment [8] of IVS shows that in massive PE circulatory failure is mediated through a profound decrease in LV preload, resulting from both pulmonary outflow obstruction and reduced LV diastolic compliance. As a result of this complex process, RV failure and ischaemia may lead to further complications, including ventricular infarction, shock, cardiac arrest, and death.

The IVS shift in CTPA was first reported by Oliver et al. [13], as an "old sign revisited", as one of the echocardiography-derived accessory signs of RVD in the course of acute PE. However, even in this first report, the authors confirm the subjective nature of this sign, both in echocardiography and CT. They also admit the obvious limitation of CT, which is the susceptibility to the motion artefacts. We also observed a significant number of heart motion artefacts, which may influence both MPR and VR methods. Although we lacked heart rate values during the examination to further analyse possible correlation, we observed the effect of motion artefacts on the discrepancy of the MPR-based assessment of IVS position, as presented in Figure 4.

Several studies have been published on the clinical implications of IVS bowing sign, with discrepancies regarding the relation with mortality, and with low kappa values for interobserver agreement.

Araoz et al. [2] reported bowing of IVS to be predictive of death due to PE with low sensitivity of about $20 \%$ and specificity of almost $90 \%$. Their report draws attention to fair interobserver variability (kappa 0.54) of IVS bowing, as well as discrepancy in the relation between IVS bowing and short-term mortality: there was a significant relation only in 2 out of 3 observers, with odds ratio of about 2 . In our study group, only 2 patients died in the hospitalisation period; therefore, we did not perform statistical analysis of IVS bowing frequency between survivors and non-survivors. In our study, the interobserver agreement of MPR-based

Table 2. Comparison of obstruction score, right ventricular/left ventricular (RV/LV) ratio, and pulmonary artery systolic pressure (PASP) values in patients with normal and abnormal position of interventricular septum (IVS) [VR (-) and VR (+), respectively] evaluated with volume rendering (VR) reconstructions

\begin{tabular}{lccccccc}
\hline & VR $(-)$ & VR (+) & p & AUC [95\% Cl] & Best cut-off & Sensitivity & Specificity \\
\hline Obstruction score [points] & $9(2-28)$ & $25(2-36)$ & $<0.001$ & $0.852[0.738-0.967]$ & 21.5 & $82 \%$ & $76 \%$ \\
RV/LV [mm] & $1.05(0.7-1.63)$ & $1.29(0.98-2.19)$ & $<0.001$ & $0.812[0.705-0.919]$ & 1.13 & $88 \%$ & $60 \%$ \\
PASP & $32(18-70)$ & $42(20-70)$ & 0.025 & $0.705[0.545-0.865]$ & 37.5 & $94 \%$ & $52 \%$ \\
\hline
\end{tabular}




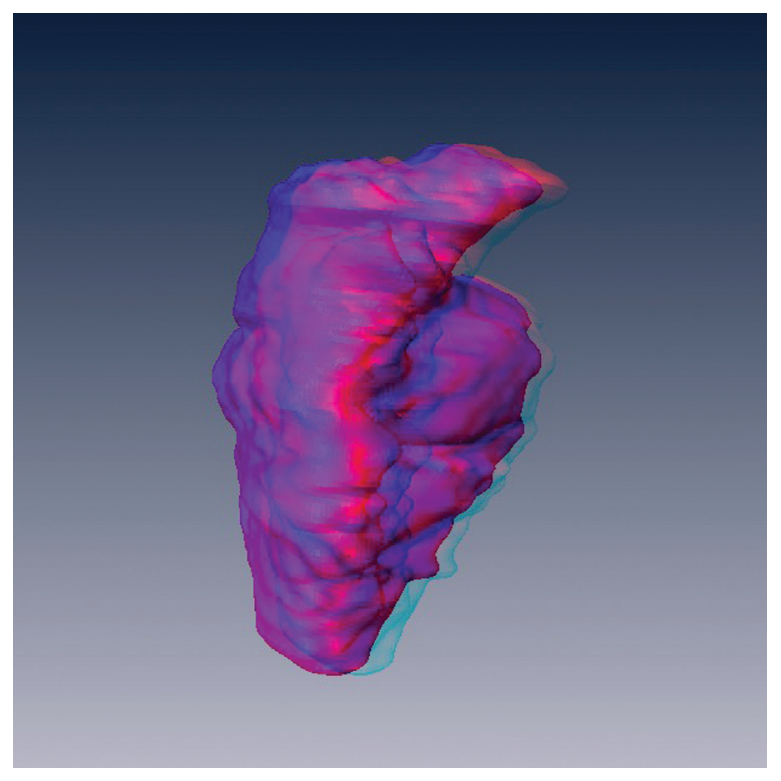

Figure 4. Volume rendering (VR) reconstruction shows significant motion artefacts, which resulted in a discrepancy in multiplanar reformation based method identification, correctly identified as interventricular septum bowing by VR-based method.

assessment of IVS position was also low. Therefore, improvement of this agreement by VR-based method seems to be a promising modification.

Kang et al. [9] report an interobserver reproducibility of describing the position of the ventricular septum as ranging between fair ( $\mathrm{kappa}=0.32,95 \% \mathrm{Cl}$ $0.090-0.551)$ to moderate $(\mathrm{kappa}=0.44,95 \% \mathrm{Cl}$ $0.220-0.685$ ), which is comparable to our results. In their population, the occurrence of IVS bowing was $18 \%$ and $8 \%$ for respective observers, which is lower than in our study as well as other reports $(23 \%$ in the study group of Araoz et al. [2]; $21 \%$ in the report by van der Meer et al. [18]). Van der Meer et al. [18] did not find a relationship between the IVS position and PE-related mortality. They observed abnormal shape of IVS in all patients with severe RVD; however, they defined RVD as an increased RV/LV ratio. A lack of correlation with mortality was also observed by Ghaye et al. [5], who observed IVS bowing in $23 \%$ of survivors and $25 \%$ of non-survivors, or more recently by Moroni et al. [12] and Heyer et al. [7].

Collomb et al. [4] observed flattening of IVS in $60 \%$ of patients with severe PE (defined on the basis of clinical status), and almost $14 \%$ of non-severe PE, while bowing of IVS occurred only in severe PE patients in $15 \%$ of cases. More recently, Zhao et al. [19] confirmed this frequency in a group of 73 patients, with abnormal position of IVS in $68 \%$ of patients with severe PE and $12 \%$ of patients with non-severe PE. In a more recent study, Kang et al. [10] showed that IVS bowing is significantly related with adverse outcomes (hazard ratio: 2.07, $p=0.007$ ), but not with early mortality.

There are several limitations to the presented study. The limited size of the study group results in a lack of correlation of IVS bowing with mortality. Furthermore, because of the retrospective character of the study, we did not have access to patients' heart rate values at the examination, which could give useful information on their influence on the artefacts. Heart motion artefacts could be avoided with ECG-gated scanning. However, Lu et al. [11] suggest that the clinical usefulness of the information gained with ECG-gating does not justify its routine use. Furthermore, our aim was to use the most widespread diagnostic modality, which is why we did not introduce the computer aided diagnosis system, which may influence the interobserver agreement [3]. Recently, interest in the application of dual-source CT for identification of PE severity has increased, also with assessment of time required for perfusion defect scoring, which appears to be less time-consuming than scoring of obstruction of pulmonary arteries [16].

\section{CONCLUSIONS}

Our paper confirms the potential of the clinical implications of IVS shift observed in patients with acute PE. Significant improvement of interobserver agreement may help improve utilisation of IVS shift; however, a method for objective identification of IVS position could possibly help increase the accuracy of this sign.

\section{ACKNOWLEDGEMENTS}

This research was supported by an luventus Plus Grant of the Ministry of Science and Higher Education, Poland, Grant Number: 0389/P01/2010/70.

\section{REFERENCES}

1. Altman DG (1991) Practical statistics for medical research. Chapman and Hall, London, UK.

2. Araoz PA, Gotway MB, Harrington JR, Harmsen WS, Mandrekar JN (2007) Pulmonary embolism: prognostic CT findings. Radiology, 242: 889-897.

3. Blackmon KN, Florin C, Bogoni L, McCain JW, Koonce JD, Lee H, Bastarrika G, Thilo C, Costello P, Salganicoff M, Joseph Schoepf U (2011) Computer-aided detection of pulmonary embolism at CT pulmonary angiography: can it improve performance of inexperienced readers? Eur Radiol, 21: 1214-1223. 
4. Collomb D, Paramelle PJ, Calaque O, Bosson JL, Vanzetto G, Barnoud D, Pison C, Coulomb M, Ferretti G (2003) Severity assessment of acute pulmonary embolism: evaluation using helical CT. Eur Radiol, 2003, 13:1508-1514.

5. Ghaye B, Ghuysen A, Willems V, Lambermont B, Gerard P, D'Orio V, Gevenois PA, Dondelinger RF (2006) Severe pulmonary embolism: Pulmonary artery clot load scores and cardiovascular parameters as predictors of mortality. Radiology, 239: 884-891.

6. Giuntini C, Di Ricco G, Marini C, Melillo E, Palla A (1995) Pulmonary embolism: epidemiology. Chest, 107 (suppl.): 3S-9S.

7. Heyer CM, Lemburg SP, Knoop H, Holland-Letz T, Nicolas V, Roggenland D (2011) Multidetector-CT angiography in pulmonary embolism-can image parameters predict clinical outcome? Eur Radiol, 21: 1928-1237.

8. Jardin F, Dubourg O, Guéret P, Delorme G, Bourdarias JP (1987) Quantitative two-dimensional echocardiography in massive pulmonary embolism: emphasis on ventricular interdependence and leftward septal displacement. J Am Coll Cardiol, 10:1201-1206.

9. Kang DK, Ramos-Duran L, Schoepf UJ, Armstrong AM, Abro JA, Ravenel JG, Thilo C. (2010) Reproducibility of CT signs of right ventricular dysfunction in acute pulmonary embolism. AJR Am J Roentgenol. 194: 1500-1506.

10. Kang DK, Thilo C, Schoepf UJ, Barraza JM Jr, Nance JW Jr, Bastarrika G, Abro JA, Ravenel JG, Costello P, Goldhaber SZ (2011) CT signs of right ventricular dysfunction prognostic role in acute pulmonary embolism. J Am Coll Cardiol Cardiovasc Imaging, 4: 841-849.

11. Lu MT, Cai T, Ersoy H, Whitmore AG, Levit NA, Goldhaber SZ, Rybicki FJ (2009) Comparison of ECG-gated versus non-gated CT ventricular measurements in thirty patients with acute pulmonary embolism. Int J Cardiovasc Imaging, 25: 101-107.

12. Moroni AL, Bosson JL, Hohn N, Carpentier F, Pernod G, Ferretti GR (2011) Non-severe pulmonary embolism: prognostic CT findings. Eur J Radiol, 79: 452-458.
13. Oliver TB, Reid JH, Murchison JT (1998) Interventricular septal shift due to massive pulmonary embolism shown by $\mathrm{CT}$ pulmonary angiography: an old sign revisited. Thorax, 53: 1092-1094.

14. Qanadli SD, El Hajjam M, Vieillard-Baron A, Joseph T, Mesurolle B, Oliva VL, Barré O, Bruckert F, Dubourg O, Lacombe $P$ (2001) New CT index to quantify arterial obstruction in pulmonary embolism: comparison with angiographic index and echocardiography. Am J Roentgenol, 176: 1415-1420.

15. Quiroz R, Kucher N, Schoepf UJ, Kipfmueller F, Solomon SD, Costello P, Goldhaber SZ (2004) Right ventricular enlargement on chest computed tomography prognostic role in acute pulmonary embolism. Circulation, 109: 2401-2404.

16. Thieme SF, Ashoori N, Bamberg F, Sommer WH, Johnson TR, Leuchte $H$, Becker A, Maxien D, Helck AD, Behr J, Reiser MF, Nikolaou K (2012) Severity assessment of pulmonary embolism using dual energy CT: correlation of a pulmonary perfusion defect score with clinical and morphological parameters of blood oxygenation and right ventricular failure. Eur Radiol, 22: 269-278.

17. Torbicki A, Perrier A, Konstantinides S, Agnelli G, Galie N, Pruszczyk P, Bengel F, Brady AJ, Ferreira D, Janssens $U$, Klepetko W, Mayer E, Remy-Jardin M, Bassand JP; ESC Committee for Practice Guidelines (2008) Guidelines on the diagnosis and management of acute pulmonary embolism: the Task Force for the Diagnosis and Management of Acute Pulmonary Embolism of the European Society of Cardiology (ESC). Eur Heart J, 29: 2276-2315.

18. Van der Meer RW, Pattynama PMT, van Strijen MJL (2005) Right ventricular dysfunction and pulmonary obstruction index at helical CT: prediction of clinical outcome during 3-month follow-up in patients with acute pulmonary embolism. Radiology, 235: 798-803.

19. Zhao DJ, Ma DQ, He W, Wang JJ, Xu Y, Guan CS (2010) Cardiovascular parameters to assess the severity of acute pulmonary embolism with computed tomography. Acta Radiol, 51: 413-419. 